\title{
On the Impact of Sharing Information in Advance Reservation Systems
}

\author{
Eran Simhon \\ Division of System Engineering \\ Boston University \\ simhon@bu.edu
}

\author{
David Starobinski \\ Division of System Engineering \\ Boston University \\ staro@bu.edu
}

\section{ABSTRACT}

Services that allow advance reservations (AR) over the Internet differ in the information provided to customers about future availability of servers. In some services, customers observe the exact number of currently available servers prior to making decisions. In other services, customers are only alerted when a few servers remain available, while there are also services in which no information whatsoever is shared about the availability of servers. Examples for the first case can be found in entertainment services, where customers are allowed to choose their seats and observe the exact number of available seats. Examples for the second case can be found in lodging reservations websites, such as Booking.com, that alert potential customers only when a few available rooms are left. Booking of airline tickets is an example of the third case where no information is provided (typically, customers can choose seats but only after buying a ticket).

In recent years, research on the impact of information on different queueing systems has emerged (see [1], for example). However, not much is known about the impact of information in systems that allow advance reservations. Our goal is to understand how different information sharing policies affect the decision of customers whether to reserve a resource in advance or not. Towards this end, we define a game, in which customers either reserve a resource in advance or avoid advance reservation and take the risk that the resource will not be available when needed. Making advance reservation is associated with a fixed cost. This cost can be interpreted as a reservation fee, as the time or resources required for making the reservation, or as the cost of financing advance payment of the service.

AR games were introduced in [2] and further investigated in [3]. In the model considered in that paper, customers are not informed about the number of available servers. In contrast, in this present work, we consider a set-up where customers can observe the state of the system prior to making a reservation.

We first study a fully-observable game. In this game, customers observe the exact number of available servers. We determine the equilibrium structure and prove the existence and uniqueness of the equilibrium.

We then consider a semi-observable game. In this game, the provider informs customers about the number of available servers only if this number is smaller or equal to some threshold. We assume that customers that are not informed

Copyright is held by author/owner(s). realize that the number of available servers is greater than that threshold and take this fact under consideration upon making their decisions. We show that, in this case, there may be multiple equilibria and the number of equilibria depends on the AR cost.

Finally, using simulations we show that, on average, the fraction of customers making AR decreases as more information is provided to the customers. More specifically, the fully observable policy yields the lowest number of reservations. In semi-observable policies, the fraction of customers making advance reservation increases as the threshold is lowered, and the best performance is achieved when no information at all is provided. Proofs of the results and more details about the simulation could be found in the working paper copy. $^{1}$

There are still many open questions remaining about the impact of sharing information on customers behavior in advance reservation services. Possible directions for further research include systems where customers have incomplete knowledge of statistics, or systems where the provider shares imprecise information.

\section{ACKNOWLEDGMENT}

This work was supported in part by the U.S. National Science Foundation under grants CNS-1117160.

\section{REFERENCES}

[1] O. Aksin, M. Armony, and V. Mehrotra, "The modern call-center: a multi-disciplinary perspective on operations management research," Production Operation Management, vol. 16, pp. 665-688, 2007.

[2] E. Simhon and D. Starobinski, "Game-theoretic analysis of advance reservation services," in Information Sciences and Systems (CISS), 2014 48th Annual Conference on. IEEE, 2014, pp. 1-6.

[3] E. Simhon and D. Starobinski, "Advance reservation games and the price of conservatism," in $A C M$ SIGMETRICS Performance Evaluation Review, vol. 42, no. 3. ACM, 2014, pp. 33-33.

\footnotetext{
${ }^{1} \mathrm{~A}$ working copy is available at http://www.bu.edu/ phpbin/cise/download.php?publication_id=1268
} 